\title{
The relevance of anti-Jo-1 autoantibodies in patients with definite dermatomyositis
}

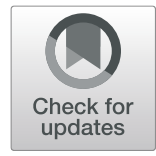

Vanessa Posener de Andrade ${ }^{1}$, Fernando Henrique Carlos De Souza', Gustavo Luiz Behrens Pinto ${ }^{1}$ and Samuel Katsuyuki Shinjo ${ }^{1,2^{*}}$ id

\begin{abstract}
Background: To assess the prevalence and clinical relevance of anti-Jo-1 autoantibodies in a representative sample of patients with definite dermatomyositis (DM).

Methods: This retrospective cohort study took place from 2005 to 2020 and assessed 118 adult patients from a tertiary center who were diagnosed with definite DM. A commercial kit was used to detect anti-Jo-1 autoantibodies.

Results: The presence of anti-Jo-1 autoantibodies was observed in 10 out of 118 (8.5\%) patients with definite DM. The following variables were comparable between individuals with and without anti-Jo-1 autoantibodies: age at diagnosis, sex, ethnicity, disease duration, follow-up period, recurrence rate, complete clinical response, death rate, and cancer incidence. There was no difference in clinical features between groups, except for an increased prevalence of "mechanic's hands," joint involvement, and lung disease, as well as a reduced occurrence of skin findings in patients positive for anti-Jo-1 autoantibodies. No anti-Jo-1-positive patients went into remission; they required greater use of glucocorticoids and immunosuppressive drugs.
\end{abstract}

Conclusions: Anti-Jo-1 positivity was found in $8.5 \%$ of patients with definite DM. This autoantibody was associated with an antisynthetase syndrome phenotype and might predict clinical outcomes in patients with definite DM.

Keywords: Autoantibodies, Dermatomyositis, Inflammatory myopathies, Myositis

\section{Introduction}

Dermatomyositis (DM) is a systemic autoimmune disease whose cardinal features include classical cutaneous findings and mostly proximal muscle weakness affecting the limbs [1-3].

The classical cutaneous manifestations of DM are Gottron's papules and heliotrope rash, but other findings such as digital ulcers, photosensitivity, calcinosis cutis, the "shawl" and "V-neck" signs, cuticular hypertrophy, periungueal erythema, and "mechanic's hands" might be present [4]. In addition, extramuscular manifestations

\footnotetext{
* Correspondence: samuel.shinjo@usp.br
${ }^{1}$ Division of Rheumatology, Faculdade de Medicina FMUSP, Universidade de

* Correspondence: samuel.shinjo@usp.br
'Division of Rheumatology, Faculdade de Medicina FMUSP, Universidade de Sao Paulo, Sao Paulo, SP, Brazil

${ }^{2}$ Disciplina de Reumatologia, Faculdade de Medicina, Universidade de Sao Paulo, Av. Dr. Arnaldo, 455, $3^{\circ}$ andar, sala 3184 - Cerqueira César, CEP: 01246-903, Sao Paulo, Brazil
}

such cardiac, gastrointestinal, joint, and lung involvement might occur [5, 6]. DM therefore has a broad spectrum of phenotypical presentations.

A variety of autoantibodies have been described in DM patients, who have myositis-associated or myositisspecific autoantibodies [6-14]. Myositis-specific autoantibodies include anti-Mi-2, anti-TIF1- $\gamma$, anti-NXP-2, anti-MDA-5, anti-SAE, and anti-aminoacyl-tRNA synthetase [6-14]. These autoantibodies are associated with different clinical phenotypes of systemic autoimmune myopathies, making them potentially useful as prognostic markers and predictors of clinical response to drug therapy [6-14].

Among anti-aminoacyl-tRNA synthetase autoantibodies, anti-Jo-1 is most commonly found in systemic autoimmune myopathies $[6,7,9,13]$. Anti-Jo-1 positivity in DM ranges from 5 to $20 \%[6,7,9,13]$ and is linked to 
the occurrence of joint involvement [9, 11-14], interstitial lung disease [10, 12-17], Raynaud's phenomenon $[13,14]$, myositis $[9,16]$, and "mechanic's hands" [6, 9, 10, 13]. However, those variables were investigated in samples comprising patients with different subtypes of systemic autoimmune myopathies, including overlap syndromes [5, 8-16]. In addition, even when only DM cases were evaluated, samples were small $[10,11,14]$, or the Bohan and Peter criteria $[1,2]$, which are restricted to muscle involvement and a narrow number of skin manifestations, were used for diagnosis. These limitations preclude an overall assessment of the relevance of anti-Jo-1 autoantibodies concerning the potential clinical features of DM.

Therefore, this study was aimed at describing the prevalence of anti-Jo-1 positivity in a representative sample of patients with definite DM and assessing the potential associations of anti-Jo-1 positivity with clinical, laboratory, and therapeutic characteristics, remission and relapse rates, and prognosis.

\section{Patients and methods}

This was a single-center, retrospective cohort study that took place from 2005 to 2020 and involved adult patients diagnosed with definite DM according to the Bohan and Peter classification criteria [1, 2], as well as the European League Against Rheumatism/American College of Rheumatology (EULAR/ACR) 2017 criteria [3]. To be included, patients had to present with pathognomonic skin rashes, as well as muscle involvement (objective muscle weakness in the upper and/or lower limbs accompanied by elevated serum creatine phosphokinase or aldolase levels). The following ancillary tests were also considered: an electromyography and nerve conduction studies suggesting an isolated myopathic process and/or a muscle biopsy suggesting an inflammatory myopathy.

Patients with clinically amyopathic DM (CADM), cancer-associated myositis, overlap syndromes as well as those with anti-OJ, anti-EJ, anti-PL-7, anti-PL-12, anti$\mathrm{SRP}$, anti-Ku, or anti-PM/Scl autoantibodies were excluded.

The following data were collected from electronic medical records containing previously parameterized and standardized information:

- General characteristics: age at diagnosis, sex, ethnicity, disease duration, and outpatient follow-up period;

- Initial and cumulative clinical manifestations: constitutional symptoms (weight loss and fever), Gottron's papules, Gottron's sign, heliotrope rash, facial erythema, Raynaud phenomenon, "shawl" sign, "V-neck" sign, cutaneous vasculitis, digital ulcers, calcinosis cutis, periungueal erythema, "mechanic's hands," dysphagia, joint involvement (arthralgia or arthritis), and lung involvement (dyspnea and chest high-resolution computed tomography - CT - imaging showing incipient interstitial lung disease, ground-glass opacities, and pulmonary fibrosis). All patients with respiratory symptoms underwent chest CT scans.

- Laboratory data: maximum serum creatine phosphokinase (reference range: 32-294 U/L) and aldolase (reference range: 1.0-7.5 U/L) levels during outpatient follow-up; blood samples collected at the beginning of the investigation of disease activity were assayed for myositis-specific (anti-Jo-1, -OJ, -EJ, -PL-7, -PL-12, -SRP, and -Mi-2) and myositisassociated (anti-Ro-52, -Ku, and -PM/Scl) autoantibodies. Myositis Profile Euroline blood test kits (Euroimmun, Germany) were used to detect the above-mentioned autoantibodies, and samples were processed as per manufacturer's instructions. The assessment of results was based on a predetermined method described elsewhere [6]. The indirect immunofluorescence assay on HEp-2 cells was used for the detection of antinuclear antibodies.

The following data were also collected at the last follow-up appointment and/or at the end of outpatient follow-up:

- Disease in remission: no evidence of disease activity for at least 6 months with no drug therapy for DM;

- Complete clinical response: no evidence of disease activity for at least 6 months with drug therapy for DM;

- Disease relapse: recurrence of clinical (muscle and/ or skin manifestations) and/or laboratory findings (elevated muscle enzymes creatine phosphokinase and aldolase) with no explanation other than disease activity;

- Drug therapy (glucocorticoids and immunosuppressants or immunomodulators);

- Occurrence of death or diagnosis of neoplasia during the follow-up period.

\section{Statistical analysis}

The Kolmogorov-Smirnov test was used to assess the distribution of continuous variables. Results are presented as means \pm standard deviations (SD) for continuous variables and as frequencies (\%) for categorical variables. Medians and interquartile ranges (75th ${ }^{-} 25$ th) were calculated for continuous variables with nonnormal distribution. Student's t-test or Mann-Whitney $\mathrm{U}$ test were used for continuous variables, and the chisquare test or Fisher's exact test were used for categorical variables in order to compare patients with and 
without of anti-Jo-1 autoantibodies. Odds ratios (OR) and $95 \%$ confidence intervals (CI) were estimated by a logistic regression model that was used to evaluate the association between anti-Jo-1 positivity and relevant covariates with statistical significance in the univariate analysis. We adopted $P$ values $<0.05$ to indicate statistical significance. SPSS Statistics, version 15.0 (Chicago, USA), was used for all analyses performed.

\section{Results}

In the present study, 152 DM patients were evaluated. Of these, 7 patients had CADM, 12 patients had cancerassociated myositis, 2 patients had overlap syndromes, and 13 patients had anti-OJ, anti-EJ, anti-PL-7, anti-PL12, anti-SRP, anti-Ku, or anti-PM/Scl autoantibodies. These patients were excluded and, therefore, 118 patients with definite DM were finally analyzed.

Anti-Jo-1 autoantibodies were detected in 10 out of 118 (8.5\%) patients with definite DM. Table 1 shows the general characteristics of DM patients with and without anti-Jo-1 autoantibodies.

A significantly increased prevalence of "mechanic's hands," as well as joint and lung involvements, and a reduced prevalence of facial erythema, "shawl" sign, and "V-neck" sign were found among patients positive for anti-Jo-1 autoantibodies compared to anti-Jo-1-negative patients (Table 1$)$.

No patients with anti-Jo-1 autoantibodies had vasculitides, digital ulcers, or calcinosis cutis (Table 1).

Regarding treatment, current use of glucocorticoids (prednisone) and immunosuppressive drugs (particularly mycophenolate mofetil) was significantly more prevalent among anti-Jo-1-positive patients compared to those without this autoantibody (Table 2).

Relapse rates, complete clinical response, and death were also comparable between the two groups. No antiJo-1-positive patients achieved disease remission or had active disease at the end of follow-up (Table 2).

In addition, all patients with anti-Jo-1 autoantibodies continued to be regularly followed up, unlike anti-Jo-1negative patients (Table 2).

All significant variables shown in Tables 1 and 2 were strongly associated with anti-Jo-1 autoantibody positivity (Table 3).

\section{Discussion}

In the present study, anti-Jo-1 positivity was observed in $8.5 \%$ of patients with definite DM, and it was associated with "mechanic's hands," as well as joint and lung involvement.

In contrast, anti-Jo-1-positive patients exhibited a decreased frequency of skin manifestations such as facial erythema, "shawl" sign, and "V-neck" sign compared to anti-Jo-1-negative patients.
The frequency of anti-Jo-1 positivity ranges from 5 to $20 \%$ in the literature $[6,7,9,10,13]$, and such wide variation occurs because of the different ethnic groups evaluated, which included Indians [10], Europeans [13], Brazilians [6], and cohorts comprising patients from multiple ethnicities [9]. In the present study, the prevalence of anti-Jo-1 positivity was $8.5 \%$ in Brazilian patients, which is within the range found in the literature. However, unlike other published research $[9,10,12-14$, 16], the presence of anti-Jo-1 autoantibodies was evaluated in a sample comprising only patients with DM in the present study. Moreover, only patients exclusively with definite DM were included, and patients with possible or probable DM, according to both the EULAR/ ACR 2017 [3] and the Bohan and Peter criteria [1, 2], were excluded. Patients with clinically amyopathic DM, myositis associated with neoplasia or other systemic autoimmune disorders, and patients with other myositisspecific (e.g., anti-OJ, -EJ, -PL-7, and -PL-12) or myositis-associated (e.g., anti-Ku and anti-PM/Scl) autoantibodies were also excluded. Although this was a retrospective study, patient data were collected from records containing previously parameterized and standardized information, including that of interest to the present study.

Although the manifestations of DM are primarily cutaneous and muscular, we observed a significant prevalence of joint involvement in our cohort. We also identified a strong association between anti-Jo-1 positivity and the occurrence of joint involvement in our sample. This association has also been found in other studies. For instance, in a large European cohort [13] comprising 1637 patients with probable or definite DM, or polymyositis (PM) according to the Bohan and Peter criteria $[1,2]$, arthritis was strongly associated with antiJo-1 positivity. In addition, in a study by Ohashi et al. [11], japanese patients with systemic autoimmune myopathies and anti-Jo-1 positivity frequently presented with joint symptoms. Such an association between arthralgia or arthritis and anti-Jo-1 positivity has also been observed among patients with multiple types of idiopathic inflammatory myopathies $[9,12,14]$. Thus, a marked influence of anti-Jo-1 autoantibodies on the phenotype of patients with DM and joint involvement can be inferred.

The cutaneous lesions known as "mechanic's hands," classically associated with antisynthetase syndrome, are characterized by hyperkeratosis, scaling, and fissuring affecting the phalanges of the hands [4]. The correlation between anti-Jo-1 autoantibodies and "mechanic's hands" has been described for Indian and Japanese patients with systemic autoimmune myopathies $[10,12]$. In a meta-analysis of 27 studies that included 3487 patients with idiopathic inflammatory myopathies, Lega et al. [9] observed that the frequency of "mechanic's hands" 
Table 1 General features of patients with dermatomyositis with versus without anti-Jo-1 autoantibodies

\begin{tabular}{|c|c|c|c|}
\hline & $\begin{array}{l}\text { Anti-Jo-1 (+) } \\
(n=10)\end{array}$ & $\begin{array}{l}\text { Anti-Jo-1 (-) } \\
(n=108)\end{array}$ & $P$ value \\
\hline Age at diagnosis (years) & $46.5 \pm 16.4$ & $43.9 \pm 15.6$ & 0.638 \\
\hline Sex: female & $5(50.0)$ & $77(71.3)$ & 0.171 \\
\hline Ethnicity: white & $9(90.0)$ & $86(79.6)$ & 0.684 \\
\hline Disease duration (years) & $5.0[1.8-8.0]$ & $7.0[4.0-12.0]$ & 0.093 \\
\hline Duration of follow-up (months) & 36.6 [18.8-95.6] & $56.0[27.5-85.5]$ & 0.804 \\
\hline Constitutional symptoms (baseline) & $9(90.0)$ & $77(71.3)$ & 0.283 \\
\hline \multicolumn{4}{|l|}{ Cumulative manifestations } \\
\hline \multicolumn{4}{|l|}{ Cutaneous involvement } \\
\hline Gottron papules & $10(100.0)$ & $108(100.0)$ & $>0.999$ \\
\hline Gottron sign & $10(100.0)$ & $108(100.0)$ & $>0.999$ \\
\hline Heliotrope eruption & $10(100.0)$ & $108(100.0)$ & $>0.999$ \\
\hline Facial erythema & $5(50.0)$ & $88(81.5)$ & 0.034 \\
\hline Raynaud phenomenon & $8(80.0)$ & $62(57.4)$ & 0.312 \\
\hline "V-neck" sign & $3(30.0)$ & $71(65.7)$ & 0.044 \\
\hline "Shawl" sign & $4(40.0)$ & $54(50.0)$ & 0.036 \\
\hline Vasculitis & 0 & $37(34.3)$ & - \\
\hline Digital ulcers & 0 & $31(28.7)$ & - \\
\hline Periungual erythema & $7(70.0)$ & $84(77.8)$ & 0.694 \\
\hline "Mechanic's hands" & $7(70.0)$ & $13(12.0)$ & $<0.001$ \\
\hline Calcinosis cutis & 0 & $7(65.0)$ & - \\
\hline Joint involvement & $8(80.0)$ & $34(31.5)$ & 0.004 \\
\hline Lung involvement & $10(100.0)$ & $33(30.6)$ & $<0.001$ \\
\hline Dyspnea & $8(80.0)$ & $30(28.7)$ & 0.002 \\
\hline Incipient interstitial lung disease & $10(100.0)$ & $28(25.9)$ & $<0.001$ \\
\hline Pulmonary fibrosis & $1(10.0)$ & $4(3.7)$ & 0.363 \\
\hline Ground-glass opacities & $6(60.0)$ & $7(6.5)$ & $<0.001$ \\
\hline \multicolumn{4}{|l|}{ Laboratory findings } \\
\hline Antinuclear antibodies & $9(90.0)$ & $81(75.0)$ & $>0.999$ \\
\hline Anti-Mi-2 & 0 & $12(11.1)$ & - \\
\hline Anti-Ro-52 & $2(20.0)$ & $22(20.4)$ & $>0.999$ \\
\hline Maximum creatine kinase levels $(U / L)$ & $4522[2173-13,500]$ & 2208 [253-8394] & 0.118 \\
\hline Maximum aldolase levels (U/L) & $50[20.6-74.8]$ & $28.1[9.0-90.5]$ & 0.617 \\
\hline
\end{tabular}

Data are shown as means \pm standard deviations, medians [interquartile ranges], or number of patients (\%)

increased by about $50 \%$ among patients with anti-Jo-1 autoantibodies compared to patients positive for other anti-aminoacyl-tRNA synthetase autoantibodies. AntiJo-1 positivity was also associated with an increased frequency of "mechanic's hands" among DM patients in the present study. This finding is also corroborated by Srivastava et al. [10], who found that patients with antisynthetase antibodies as a group and those with anti-Jo-1 antibodies alone had significantly more "mechanics' hands," while this association was not noted with other non-Jo-1 antisynthetase autoantibodies.
As for the other DM skin lesions, we observed a reduced prevalence of facial erythema, "shawl" sign, and "V-neck" sign, and no digital ulcers or calcinosis cutis among our anti-Jo-1-positive patients. Thus, this autoantibody might act as a protective factor against these cutaneous manifestations; however, further research is needed to investigate this hypothesis.

Interstitial lung disease is associated with increased morbidity and mortality in patients with idiopathic inflammatory myopathies $[18,19]$. We observed a strong association between anti-Jo-1 autoantibodies and lung involvement, including clinical (dyspnea) and 
Table 2 Current treatment, relapsing, disease status, death, neoplasia, and follow-up of patients with dermatomyositis

\begin{tabular}{|c|c|c|c|}
\hline & $\begin{array}{l}\text { Anti-Jo-1 (+) } \\
(n=10)\end{array}$ & $\begin{array}{l}\text { Anti-Jo-1 (-) } \\
(n=108)\end{array}$ & $P$ value \\
\hline \multicolumn{4}{|l|}{ Treatment } \\
\hline \multicolumn{4}{|l|}{ Prednisone } \\
\hline Current use & $7(70.0)$ & $32(29.6)$ & 0.015 \\
\hline Current dose (mg/day) & 15 [5-40] & $10[5-40]$ & 0.788 \\
\hline Immunosuppressive drugs & $10(100.0)$ & $63(58.3)$ & 0.013 \\
\hline Methotrexate & $3(30.0)$ & $27(25.0)$ & 0.715 \\
\hline Azathioprine & $1(10.0)$ & $22(20.4)$ & 0.684 \\
\hline Mycophenolate mofetil & $5(50.0)$ & $14(13.0)$ & 0.010 \\
\hline Cyclosporine & $1(10.0)$ & $6(5.6)$ & 0.471 \\
\hline Leflunomide & $1(10.0)$ & $7(6.5)$ & 0.519 \\
\hline Intravenous immunoglobulin & $1(10.0)$ & $1(0.9)$ & 0.163 \\
\hline Rituximab & $2(20.0)$ & $11(10.2)$ & 0.307 \\
\hline Disease relapse & $7(70.0)$ & $56(51.9)$ & 0.508 \\
\hline \multicolumn{4}{|l|}{ Current disease status } \\
\hline Disease remission & 0 & $38(35.2)$ & - \\
\hline Complete clinical response & $2(20.0)$ & $32(29.6)$ & 0.722 \\
\hline Disease activity & 0 & $10(9.3)$ & - \\
\hline Death & $1(10.0)$ & $9(8.3)$ & $>0.999$ \\
\hline Neoplasia & 0 & 0 & - \\
\hline Follow-up & $10(100.0)$ & $58(53.7)$ & 0.005 \\
\hline
\end{tabular}

Data are shown as medians [interquartile ranges] or number of patients (\%)

radiographic abnormalities on high-resolution $\mathrm{CT}$ imaging suggestive of interstitial lung disease. Anti-Jo-1 positivity is indeed associated with interstitial lung disease as defined by imaging findings alone [6] or together with abnormal pulmonary function testing [10, 12-17]. In our cohort, not all patients underwent pulmonary function testing, and therefore we did not include those

Table 3 Univariate analysis of relevant variables in anti-Jo-1positive patients

\begin{tabular}{lll}
\hline & OR & $\mathbf{9 5 \% ~ C l}$ \\
\hline Facial erythema & 0.37 & $0.18-0.77$ \\
"V-neck" sign & 0.48 & $0.30-0.78$ \\
"Shawl" sign & 0.83 & $0.47-1.43$ \\
"Mechanic's hands" & 5.82 & $3.0-11.16$ \\
Joint involvement & 2.92 & $1.68-3.85$ \\
Lung involvement & 3.27 & $2.46-4.35$ \\
$\quad$ Dyspnea & 2.88 & $1.87-4.45$ \\
$\quad$ Incipient interstitial lung disease & 3.86 & $2.80-5.31$ \\
$\quad$ Ground-glass opacities & 9.26 & $3.85-22.25$ \\
Prednisone (current use) & 2.36 & $1.43-3.89$ \\
Mycophenolate mofetil & 3.86 & $1.75-8.49$ \\
Follow-up & 1.86 & $1.56-2.22$ \\
\hline
\end{tabular}

$\mathrm{Cl}$ confidence interval, $O R$ odds ratio data in the present study. However, previous research $[6$, 10, 12-17] has examined heterogeneous samples of patients with systemic autoimmune myopathies, and also has included cases of probable DM or PM, defined according to the Bohan and Peter criteria $[1,2]$ as well as overlap syndromes $[10,16]$. Furthermore, no additional analysis to exclude potential cases of antisynthetase syndrome [10, 12, 13, 15, 17], which might also affect the lungs [20-22], has been performed.

We also found that anti-Jo-1 positivity was associated with lung involvement in patients with definite DM. We excluded patients who were positive for other anti-aminoacyltRNA synthetase autoantibodies, anti-Ku, and anti-PM/Scl to avoid biasing the interpretation of our data, as they are also associated with lung involvement [20, 23, 24].

As for other autoantibodies, anti-Ro-52 is a myositisassociated antibody and is commonly related to anti-Jo-1 positivity $[6,9,16,25]$. The co-occurrence of anti-Ro-52 and anti-Jo-1 positivity was reported by Marie et al. [25] to be prevalent in patients with systemic autoimmune myopathies, suggesting that the simultaneous presence of these autoantibodies is associated with an increased risk of severe interstitial lung disease, more severe myositis, joint involvement, and neoplasia in patients older than 50 years. A potential explanation for the fact that anti-Ro-52 positivity was not associated with the 
occurrence of anti-Jo-1 autoantibodies in the present study is the small size of our sample.

Anti-Mi-2 positivity is known to be associated with the classical DM skin findings [25-27], to be a protective factor for lung involvement $[25,26]$, and a marker of high rates of remission [26] and response to glucocorticoid therapy $[25,26]$. No anti-Jo-1-positive patients in our sample were found to be positive for anti-Mi-2 autoantibodies, which reaffirms the reports in the literature suggesting myositis-specific antibodies are mutually exclusive [25].

Concerning treatment, patients with anti-Jo-1 autoantibodies were found to be more frequently on glucocorticoids and immunosuppressive drugs when compared to those negative for this autoantibody. Consequently, no anti-Jo-1-positive patients had achieved disease remission at the time of their last medical evaluation, whereas one third of those negative for this autoantibody had gone into clinical remission. Patients in remission are referred to follow-up at primary or secondary care facilities as per our local protocol, which is why approximately half of the anti-Jo-1-negative DM patients had not been receiving follow-up treatment at the time.

No cases of neoplasia were observed during follow-up, but $10 \%$ of patients died, with no difference between the groups. Relapse rates were also similar between groups.

As a major limitation of our study, we had a small number of patients with anti-Jo-1 autoantibodies, and so our results should be interpreted with caution. Moreover, since this is a retrospective study, we did not have complete data available, such as serum levels of creatine phosphokinase and aldolase at disease onset or diagnosis or serum levels of the other muscle enzymes (aspartate aminotransferase, alanine aminotransferase, lactic dehydrogenase).

\section{Conclusions}

Anti-Jo-1 autoantibodies were detected in $8.5 \%$ of DM patients and were correlated with an increased frequency of lung and joint involvement as well as "mechanic's hands," resembling the clinical presentation of antisynthetase syndrome. In contrast, anti-Jo-1 positivity was not correlated with cutaneous manifestations such as the "shawl" sign, "V-neck" sign, vasculitides, and digital ulcers. Anti-Jo-1 autoantibodies were not found to affect relapse rates, but no anti-Jo-1-positive patients went into remission, so they continued to require regular outpatient follow-up. Our study emphasizes the relevant role of anti-Jo-1 autoantibodies in the phenotypical characterization of patients with definite DM, as well as in disease progression, by excluding an important confounder present in other research, which is the inclusion of patients with collagen diseases and different systemic autoimmune myopathies, in addition to patients with antisynthetase syndrome itself. Our data also favor the tendency to characterize DM patients into more homogenous groups according to their phenotypical presentation related to myositis-specific antibodies.

\section{Abbreviations \\ ACR: American College of Rheumatology; CADM: Clinically amyopathic dermatomyositis; CT: Computed tomography; DM: Dermatomyositis; EULAR: European League Against Rheumatism}

\section{Acknowledgments}

Not applicable.

\section{Authors' contributions}

All authors contributed equally to write and review the manuscript. The author(s) read and approved the final manuscript.

\section{Funding}

Fundação de Amparo à Pesquisa do Estado de São Paulo (FAPESP) \#2014/ 09079-1, Conselho Nacional de Desenvolvimento Científico e Tecnológico (CNPq) \#303379/2018-9 and Faculdade de Medicina da USP - SP to SKS.

\section{Availability of data and materials}

Not applicable.

Ethics approval and consent to participate

This study was approved by the local ethics committee (no. 0114/11).

Consent for publication

Not applicable.

\section{Competing interests}

All authors declare that they have no conflicts of interest.

Received: 6 December 2020 Accepted: 10 February 2021

Published online: 19 February 2021

\section{References}

1. Bohan A, Peter JB. Polymyositis and dermatomyositis (first of two parts). N Engl J Med. 1975;292:344-7.

2. Bohan A, Peter JB. Polymyositis and dermatomyositis (second of two parts). N Engl J Med. 1975;292:403-7.

3. Lundberg IE, Tjärnlund $A$, Bottai $M$, Werth VP, Pilkington $C$, de Visser $M$, International Myositis Classification Criteria Project Consortium, the Euromyositis Register, and the Juvenile Dermatomyositis Cohort Biomarker Study and Repository (UK and Ireland), et al. 2017 European league against rheumatism/American college of rheumatology classification criteria for adult and juvenile idiopathic inflammatory myopathies and their major subgroups. Arthritis Rheumatol. 2017;69:2271-82.

4. Muro Y, Sugiura K, Akiyama M. Cutaneous manifestations in dermatomyositis: key clinical and serological features: a comprehensive review. Clinic Rev Allerg Immunol. 2016:51:293-302.

5. Souza FH, Barros TB, Levy-Neto M, Shinjo SK. Adult dermatomyositis: experience of a Brazilian tertiary care center. Rev Bras Reumatol. 2012;37: 264-7.

6. Cruellas MG, Viana VS, Levy-Neto M, Souza FH, Shinjo SK. Myositis-specific and myositis-associated autoantibody profiles and their clinical associations in a large series of patients with polymyositis and dermatomyositis. Clinics. 2013;68:909-14

7. Wolstencroft PW, Fiorentino DF. Dermatomyositis clinical and pathological phenotypes associated with myositis-specific autoantibodies. Curr Rheumatol Rep. 2018;20:28.

8. Alenzi FM. Myositis specific autoantibodies: a clinical perspective. Open Access Rheumatol. 2020;12:9-14.

9. Lega JC, Fabien N, Reynaud Q, Durieu I, Durupt S, Dutertre M, et al. The clinical phenotype associated with myositis-specific and associated autoantibodies: a meta-analysis revisiting the so-called antisynthetase syndrome. Autoimmun Rev. 2014;13:883-91. 
10. Srivastava P, Dwivedi S, Misra R. Myositis-specific and myositis-associated autoantibodies in Indian patients with inflammatory myositis. Rheumato Int. 2016;36:935-43.

11. Ohashi K, Sada KE, Nakai Y, Matsushima S, Asano Y, Hayashi K, et al. Cluster analysis using anti-aminoacyl-tRNA synthetases and SS-A/Ro52 antibodies in patients with polymyositis/dermatomyositis. J Clin Rheumatol. 2019;25:246-51.

12. Yamasaki Y, Satoh M, Mizushima M, Okazaki T, Nagafuchi H, Ooka S, et al. Clinical subsets associated with different anti-aminoacyl transfer RNA synthetase antibodies and their association with coexisting anti-Ro52. Mod Rheumatol. 2016;26:403-9.

13. Betteridge Z, Tansley S, Shaddick G, Chinoy H, Cooper RG, New RP, et al. Frequency, mutual exclusivity and clinical associations of myositis autoantibodies in a combined European cohort of idiopathic inflammatory myopathy patients. J Autoimmun. 2019;101:48-55.

14. Mielnik P, Wiesik-Szewczyk E, Olesinska M, Chwalinska-Sadowska H, Zabek J. Clinical features and prognosis of patients with idiopathic inflammatory myopathies and anti-Jo-1 antibodies. Autoimmunity. 2006;39:243-7.

15. Zhang L, Wu G, Gao D, Liu G, Pan L, Ni L, et al. Factors associated with interstitial lung disease in patients with polymyositis and dermatomyositis: a systematic review and meta-analysis. PLoS One. 2016;11:e0155381.

16. Hamaguchi Y, Fujimoto M, Matsushita T, Kaji K, Komura K, Hasegawa M, et al. Common and distinct clinical features in adult patients with antiaminoacyl-tRNA synthetase antibodies: heterogeneity within the syndrome. PLoS One. 2013;8:e60442.

17. Li S, Ge Y, Yang H, Wang T, Zheng X, Peng Q, et al. The spectrum and clinical significance of myositis-specific autoantibodies in Chinese patients with idiopathic inflammatory myopathies. Clin Rheumatol. 2019;38:2171-9.

18. Marie I, Haltron PY, Dominique S, Cherin P, Mouthron L, Menard JF. Short term and long term outcomes of interstitial lung disease in polymyositis and dermatomyositis: a series of 107 patients. Arthritis Rheum. 2011;63:3439-47.

19. Johnson C, Pinal-Fernandez I, Parikh R, Paik J, Albayda J, et al. Assessment of mortality in autoimmune myositis with and without associated interstitial lung disease. Lung. 2016;194:733-7.

20. Souza FH, Cruellas MG, Levy-Neto M, Shinjo SK. Anti-synthetase syndrome: anti-PL-7, anti-PL-12 and anti-EJ. Rev Bras Reumatol. 2013;53:352-7.

21. Baccaro ACCD, Behrens Pinto GL, Carboni RCS, Shinjo SK. The clinical manifestations at the onset of antisynthetase syndrome: a chameleon with multiple faces. Reumatismo. 2020;72:86-72.

22. Cavagna L, Trallero-Araguás E, Meloni F, Cavazzana I, Rojas-Serrano J, Feist E, et al. Influence of antisynthetase antibodies specificities on antisynthetase syndrome clinical spectrum time course. J Clin Med. 2019;8:2013.

23. Alves SP, Silva MG, Borges IBP, Shinjo SK. Patients with pure dematomositis/ polymyositis and anti-PM/Scl autoantibody resembling anti-synthetase syndrome. MedicalExpress. 2018;8:mo18002.

24. Yamasaki Y, Yamada H, Nozaki T, Akaogi J, Nichols C, Lyons R, et al. Unusually high frequency of autoantibodies to PL-7 associated with milder muscle disease in Japanese patients with polymyositis/dermatomyositis. Arthritis Rheum. 2006:54:2004-9.

25. Marie I, Hatron PY, Dominique S, Cherin P, Mouthon L, Menard JF, et al. Short-term and long-term outcome of anti-Jo1-positive patients with antiRo52 antibody. Semin Arthritis Rheum. 2012;41:890-9.

26. Hamaguchi Y, Kuwana M, Hoshino K, Hasegawa M, Kaji K, Matsushita T, et al. Clinical correlations with dermatomyositis-specific autoantibodies in adult Japanese patients with dermatomyositis: a multicenter cross-sectional study. Arch Dermatol. 2011;147:391-8.

27. Dos Passos Carvalho MIC, Shinjo SK. Frequency and clinical relevance of anti-Mi-2 autoantibody in adult Brazilian patients with dermatomyositis. Adv Rheumatol. 2019;59:27.

\section{Publisher's Note}

Springer Nature remains neutral with regard to jurisdictional claims in published maps and institutional affiliations.

\section{Ready to submit your research? Choose BMC and benefit from:}

- fast, convenient online submission

- thorough peer review by experienced researchers in your field

- rapid publication on acceptance

- support for research data, including large and complex data types

- gold Open Access which fosters wider collaboration and increased citations

- maximum visibility for your research: over $100 \mathrm{M}$ website views per year

At BMC, research is always in progress.

Learn more biomedcentral.com/submissions 